\title{
On Modelling and Solving Heterogeneous Vehicle Routing Problem with Multi-Trips and Multi-Products
}

\author{
Fran Setiawan ${ }^{1 *}$, Nur Aini Masruroh ${ }^{2 *}$, Zita Iga Pramuditha ${ }^{2}$
}

\begin{abstract}
Vehicle routing problem (VRP) is a model to determine an optimal routing plan for a fleet of homogeneous vehicles to serve a set customer which some operational constraints are satisfied. In most practical distribution problems, customer demands are served using a heterogeneous fleet of vehicles. This kind of VRP is called Heterogeneous Vehicle Routing Problem (HVRP). HVRP has evolved into a vibrant research area because of its practical. There were many studies of rich extensions of the standard HVRP. This research aims to enrich the extensions of HVRP which is motivated by a real case in one of a pharmacy distribution company in Indonesia which is delivered multi-products to its 55 customers by allowing some vehicles which have a small capacity to perform multi-trips. This problem is called Heterogeneous Vehicle Routing Problem with Multi-Trips and Multi-Products (HVRPMTMP). The mixed-integer linear programming is developed based on four-index vehicle flow formulation. The model can be used generally in the same context of the distribution problem. HVRPMTMP is generally NP-Hard problem, so the computational time using branch and bound in LINGO 16.0 is increasing exponentially by increasing the number of customers. Genetic algorithm is proposed to solve the real case. The result of the proposed GA can reduce the total cost from Rp 352540.6,- to Rp 180555,or $48.78 \%$ from the current company policy.
\end{abstract}

Keywords: Heterogeneous Vehicle Routing Problem; Multi-Trips; Multi-Products; Mixed Integer Linear Programming; Genetic Algorithm.

\section{Introduction}

Transportation cost can be reduced in several ways. One of them is by optimizing the sequence of vehicle route. The classical vehicle routing problem (VRP) aims to design optimal delivery routes where each vehicle has the same characteristics (capacity), each vehicle only travels one route with only one depot (Braekers et al. [1]). The VRP aims to determine routes of given vehicle fleet at minimum cost (Toth and Vigo [2]).

Since the VRP problem was first introduced by Dantzig and Ramzer [3], hundreds of papers were devoted to the exact and approximate solution of the many variants of VRP (Baldacci et al. [4]). There exists a rich literature on the VRP and its variants. The surveys are conducted by Cordeau et al. [5]; Laporte [6]; Braekers et al. [1]; and the books by Golden et al. [7] and Toth and Vigo [2]. In classical VRP which is called Capacitated Vehicle Routing Problem (CVRP), the fleet of a vehicle is homoge neous (identical) while in most practical distribution problems, customer demands are served by heterogeneous fleet of vehicles.

\footnotetext{
${ }^{1}$ Faculty of Industrial Technology, Department of Industrial Engineering, Universitas Katolik Parahyangan, JL. Ciumbuleuit 94, Bandung 40141, Indonesia Email: fransetiawan@unpar.ac.id.

2 Faculty of Engineering, Department of Mechanical and Industrial Engineering, Universitas Gadjah Mada, JL. Grafika 2, Jogjakarta 55284, Indonesia. Email: aini@ugm.ac.id; zita.pramuditha@gmail.com

* Corresponding author
}

This problem is called Heterogeneous Vehicle Routing Problem (HVRP) (Koç et al. [8]).

HVRP generally considers a limited or an unlimited fleet of capacitated vehicles, where each vehicle has a fixed cost, in order to serve a set of customers with known demands. Two major HVRP problems are the Fleet Size and Mix Vehicle Routing Problem (FSM) which works with an unlimited heterogeneous fleet and the Heterogenous Fleet Vehicle Routing Problem (HFVRP) in which the vehicle is limited (Koç et al. [8]).

HVRP, which was introduced some 30 years ago, has evolved into a rich research area because of its practical. The number of research in HVRP area are increasing in the last decade for both FSM and HFVRP. Several versions of the problem have been studied, and its applications are encountered in many settings. Research in standard HVRP and FSM focuses on lower bounding techniques, heuristics and metaheuristic for solving HVRP. Over the years, most of the research effort has shifted toward the study of rich extensions of the standard HVRP, such as time windows, multiple depots, external carriers, pickup and deliveries, multi-trip, backhaul and green HVRP. There still exist numerous research opportunities on these rich extensions (Koç et al. [8]).

In order to enrich the extensions of HVRP and inspired by a real case in pharmacy distribution 
company in Indonesia that has heterogenous fleet in delivering their multi-products to their customer and it has kind of vehicles with small capacity. These small capacity vehicles are possible to make more than one trip in their workday in order to maximize their utilization. This condition is called multi-trips VRP (Gendreau et al. [9]). The multi-trips is indicated by allowing the vehicle to perform more than one route during a planning horizon (Suprayogi and Priyandari [10]). Due to the possibility to assign several routes to the same vehicle, multi-trips VRP can make using fewer vehicles (Taillard et al. [11]). The fixed cost of vehicles can be saved by using fewer vehicles (Huang and Lee [12]). The multi-trips VRP has gained research interest for the recent year is the development of new distribution schemes in cities. The new scheme is related to congestion and pollution to study new distribution scheme to increase city livability. The infrastructure of the city often forces the final deliveries to be performed using a small size vehicle. (Cataruzza et al. [13]).

Research in HVRP with multi-trips (HVRPMT) has been conducted by Prins [14], Sexias and Mendez [15], Cruz et al. [16] and Coelho et al. [17]. Prins [14] developed a heuristic to minimize the total duration of trips and to minimize the number of required vehicles as a secondary objective. There was no mathematical model and not involving the cost of vehicles. The total duration of every vehicle is limited to working hour per day. Sexias and Mendez [15] tackled HVRPMTMP with time windows and restricted to driver hours using column generation procedure to find dual bounds. The maximum number of routes in a workday is predetermined for convenience purpose. The goal is to determining the workday of each vehicle in the fleet to minimize the total cost of the distribution operation from a single depot using vehicle variable cost. Cruz et al. [16] proposed HVRPMT with maximum route distance in which all vehicles must be used at least once before allowing multi-trips. The fixed costs are ignored, and the routing cost is vehicle-independent. In this research, the maximum demand for a given customer is greater than the minimum vehicle capacity. Coelho et al. [17] proposed HVRPMT with docking constraints in which some vehicles are unable to serve some particular customers. They proposed a trajectory search heuristic called GILS-VND that combines iterated local search (ILS), greedy randomized adaptive search procedure (GRASP) and variable neighborhood descent (VND) procedures. The objective function was minimizing vehicles fixed cost and distance-based cost.

The researches above are minimizing the travel time and the number of vehicles (Prins [14]), minimizing total vehicle variable cost (Sexias and Mendez [15] and Cruz et al. [16]), minimize vehicle fixed cost and distance-based cost (Coelho et al., [17]). There is no mathematical formulation in Prins [14], Cruz et al. [16], and Coelho et al. [17]. Mathematical modelling was developed in integer programming formulation with variable vehicle cost, time windows and driver work hour in Sexias and Mendez [15]. To our best knowledge, the mathematical formulation of HVRPMT with a fixed cost and variable cost has never been developed before. This fixed cost mainly occurs when the vehicle is not owned by the company, and the vehicle is performed by the third party (the cost to rent vehicle). The number of vehicles can be reduced since the fixed cost is also reduced by performing multi-trips.

In this research, we modeled a real case in an Indonesia' pharmacy distribution company. This company delivers multi-products. Moreover, we also propose a mathematical model of Heterogeneous Vehicle Routing Problem with Multi-Trips and Multi-Products (HVRPMTMP) with fixed and variable vehicle cost to solve that problem.

HVRPMTMP is a natural generalization of VRP, so HVRPMTMP is an NP-hard problem. Since it is an NP-hard problem, a metaheuristic method is necessary to solve the problem in reasonable computational time. Metaheuristic can carry out a more thorough search in the solution space, and it can produce high-quality solution consistently, despite it needs a longer computational time than heuristic (Cordeau et al. [5]).

\section{Methods}

In this section, we present study literature of related work on heterogeneous vehicle routing problem with multi-trips and heterogeneous vehicle routing problem with multi-products. We also present the general ideas of this research.

\section{Related Work on Heterogeneous Vehicle Routing Problem with Multi-trips}

Researches in heterogeneous vehicle routing problem with multi-trips (HVRPMT) have been conducted by Prins [14], Sexias and Mendes [15] and Cruz et al. [16]. Prins [14] proposed a heterogeneous vehicle routing problem with multi-trips with a fixed number of vehicles. He developed a heuristic to minimize the total duration of trips and to minimize the number of required vehicles as secondary objectives. There is no mathematical model and not involving the cost of vehicles. The total duration of every vehicle is limited to working hour per day. He tried several heuristics and chose the very efficient one to give outstanding initial solutions for a tabu 
search method. He used a real case of a French manufacturer of furniture with 775 destination stores.

Sexias and Mendes [15] researched heterogeneous vehicle routing problem with multi-trips with time windows and driver work hours. A mathematical model was made denoted as integer programming based on arc flow variables. The maximum number of routes in a workday is predetermined for convenience purpose. The goal is to determining the workday of each vehicle in the fleet to minimize the total cost of the distribution operation from a single depot using vehicle variable cost. They used constructive heuristic and tabu search to find a good valid integer solution. To evaluate the performance of the heuristic, a column generation algorithm with state of the art techniques was presented that was able to generate excellent dual bounds. The column generation was solved by a particular dynamic programming algorithm for the elementary shortest path problem with resource constraints.

Cruz et al. [16] proposed HVRPMT with maximum route distance in which all vehicles must be used at least once before allowing multi-trips. The fixed costs are ignored, and the routing costs are vehicleindependent. In this research, the maximum demand of a given customer is greater than the minimum vehicle capacity. This work was motivated by a real case problem of a large-size distribution company operating more than 370 stores in the Northeast of Spain. The multi-trips were performed whenever the demand in a given time period is greater than its fleet capacity. To solve this problem, they proposed a hybrid algorithm that combined a randomized Clarke and Wright's Savings heuristic and three local search methods: 2-opt, a temporary memory of the best routes found, and a splitting technique.

Coelho et al. [17] studied HVRPMT that considers docking constraint in which some vehicles are unable to serve some particular customers. This study was inspired by a real case of a large distribution company. The objective function was to minimize vehicle fixed cost, distance-based cost and a cost per customer visited. They proposed a trajectory search heuristic called GILS-VND that combined iterated local search (ILS), greedy randomized adaptive search procedure (GRASP) and Variable Neighborhood Descent (VND) procedures.

\section{Related Work on Heterogeneous Vehicle Routing Problem with Multi-products}

Researches in heterogeneous vehicle routing problem with multi-products (HVRPMP) have been conducted by Prive et al. [18] and Cruz et al. [19]. Prive et al. [18] proposed a heterogeneous vehicle routing problem with multi-products arising in soft drink distribution. The vehicles must deliver goods and recyclable pickup materials at customer locations. Their unit weight measures products and unit volume restricted to vehicle volume capacity and vehicle weight capacity. The problem was modeled as a mixed-integer program and proposed three heuristics, a constructive heuristic and two petal based heuristics.

Cruz et al. [19] conducted research in heterogeneous vehicle routing problem with multi-products with time windows. Products are also measured by its weight and volume restricted to vehicle volume capacity and vehicle weight capacity, which is same as what has been done by Prive et al. [18] above. The problem was modeled with integer programming. To solve this problem, they proposed a procedure based on ant colony optimization with two-pheromone trail strategy combined with tabu search.

\section{General Ideas on This Research}

This research proposes the new extension of a heterogeneous vehicle routing problem with multitrips and multi-products (HVRPMTMP) with fixed and variable vehicle cost. A real case motivates this research is one of a pharmacy distribution company in Indonesia. The company has to deliver its multiproducts to 55 customers scattered in Yogyakarta, Indonesia with heterogeneous kind of vehicles and there are vehicles with small capacities that can make more than one route in its working day in order to maximize their utilization. The objective is to minimize total cost. Two scenarios are made in this research. The first scenario is if the vehicles are owned by the company, so only the variable cost is considered. The second scenario is if the vehicles are not owned by the company, so there is a fixed cost of every vehicle that is different from vehicle type. This fixed cost is the cost to rent a vehicle which performed by the third party. Vehicle variable cost is dependent on the vehicle type that is obtained by fuel cost per minute. The mathematical model is developed using mixed-integer linear programming (MILP) based on four-index vehicle flow formulation, which was proposed by Cattaruzza et al. [13]. This model is solved using branch and bound in LINGO 16.0 solver and genetic algorithm. In this research, we use travel time data between customers rather than the distance between customers because travel time is capturing more the real traffic condition in the city due to congestion.

\section{Research Method}

Our research method is begun with study the real case distribution problem in one of a pharmacy distribution company in Indonesia and do literature study about previous research in HVRP and find the 
extension possibility in HVRP problem by considering that real case. Then we make a system description of the problem. After studying the system description, then we develop a mathematical model by studying the mathematical model in previous researches which related to this problem and make an adjustment on them based on the characterization of the studied system. A mathematical model that has been developed is then tested to a simple case to check whether the developed model is valid. Model is solved using branch and bound in LINGO 16.0 solver and examine the result. Output verification is done by examining the output of the mathematical model from the LINGO 16.0 whether it exceeds the constraints. The real case is solved with an exact method (Branch and Bound) in LINGO 16.0 solver. HVRPMTMP is NP-Hard so firstly we try to use a small number of customers first to know how far the exact method can handle the problem as the number of customers increase. If the case can not be solved exactly using LINGO 16.0 in reasonable computational time, then metaheuristic method is proposed to solve the problem. We proposed a genetic algorithm to help in solving a real case. After the genetic algorithm is developed, the GA algorithm is compared to the exact solution of small problem size from LINGO 16.0. If the result from the exact solution and GA are the same for small problem size, the algorithm is verified. Then the result between our proposed GA is compared with the current company policy and then analyzed.

\section{Results and Discussions}

\section{Problem Definition and Notation}

The system studied in this research is a distribution planning system which company has to distribute its multi-products and has heterogeneous vehicles (different types and capacities) to serve its customers in one period of a working day. The vehicles are allowed to make more than one trip as long as they do not exceed the working day. The sum of the customer demand visited by a vehicle in one route does not exceed the vehicle capacity. All customers must be visited, and each customer is visited exactly once. Customer demand is delivered in full supply (there is no split delivery) and deterministic. All deliveries are started from the depot and ended at the depot. Customer demand is calculated as volume demand based on the unit volume of the product and the quantity of the product demanded by the customer. The illustration of the problem studied in this research is shown in Figure 1.

Figure 1 shows that there are three types of vehicles (blue, red and green). Vehicle blue is assigned two trips to visited customer 12 , and 7 then go back to the depot and visited customer 3 and 10. Vehicle red is assigned one trip to visited customer 2,4 and 9 .

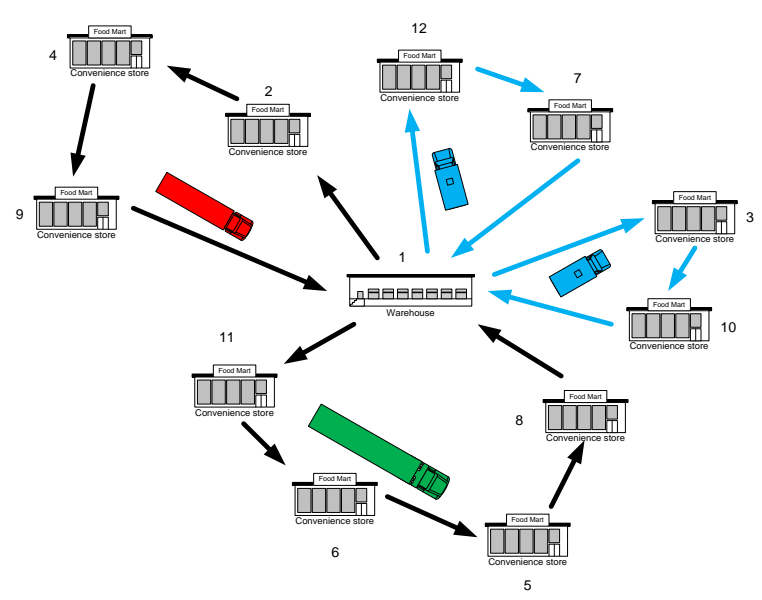

Figure 1. The illustration of the problem

Vehicle green is assigned one trip to visited customer $11,6,5$ and 3.

In graph, HVRPMTMP is defined as a complete graph $G=(N, A)$ where $N=\{0,1,2, \ldots, N\}$ is the set of nodes, node 0 corresponds to depot and $A=\{(i, j): i, j \in N$ and $i \neq j$ is the set of arcs. Customers set is denoted by $N_{0}=N \backslash\{0\}$; each member has the total demand $Q_{i}$. Travel time from node $i$ to $j$ is denoted as $T_{i j}$. $A_{i}$ is the quantity of product $p$ which is demanded by customer $i$, while $B_{p}$ is a unit volume of product $p . k=(1,2, \ldots, K)$ is the set of vehicles with heterogeneous capacity. Capk is the capacity of vehicle $k . V_{k}$ is the variable cost of using vehicle $k$, and $F_{k}$ is the fixed cost of using vehicle $k . T_{\max }$ is working hour per day. $r=$ $\{1,2, \ldots R)$ is the set of routes of each vehicle. $N R K$ is the total number of vehicles available times the total routes available. Total routes are set arbitrary and assumed the same for all vehicles since there is a working hour restriction that limits the number of routes a vehicle can travel. $C_{i j k r}$ is the cost of transportation from customer $i$ to customer $j$ using vehicle $k$ at route $r$ that is obtained from $T_{i j}$ multiplied by $V_{k}$. $Y_{i k r}$ is 1 if customer $i$ is visited by vehicle $k$ at route $r, 0$ otherwise. $U_{i k r}$ is a load of the vehicle $k$ at route $r$ after visiting customer $i . X_{i j k r}$ is 1 if from customer $i$ to customer $j$ is assigned using vehicle $k$ at route $r$.

\section{Mathematical Model}

The mathematical model for HVRPMTMP is constructed as follow

Objective function:

$\operatorname{Min} \sum_{k=1}^{K} F_{k} Y_{0 k 0}+\sum_{i=0}^{N} \sum_{j=0}^{N} \sum_{k=1}^{K} \sum_{r=1}^{R} C_{i j k r} X_{i j k r}$

Subject to:

$\sum_{k=1}^{K} \sum_{r=1}^{R} Y_{i k r}=1 \quad \forall i \in N \backslash\{0\}$

$\sum_{k=1}^{K} \sum_{r=1}^{R} Y_{0 k r} \leq N R K$

$\sum_{j=0}^{N} X_{i j k r}=Y_{i k r} \forall i \in N ; k=1, \ldots, K ; r=1, \ldots, R$ 


$$
\begin{aligned}
& \sum_{\substack{j=0 \\
i \neq j}}^{N} X_{j i k r}=Y_{i k r} \forall i \in N ; k=1, \ldots, K ; r=1, \ldots, R \\
& \sum_{p=1}^{P} A_{i p} B_{p}=Q_{i} \quad \forall i \in N \\
& \sum_{j=1}^{N} X_{0 j k r} \geq \sum_{j=1}^{n} X_{0 j k r+1} \quad \forall k=1, \ldots, K \\
& \quad r=1, \ldots, R-1 \\
& \sum_{i=1}^{N} Q_{i} Y_{i k r} \leq \operatorname{Cap}_{k} \forall k=1, \ldots, K ; r=1, \ldots, R \\
& Q_{i} \leq U_{i k r} \leq \operatorname{Cap}_{k} \quad \forall i \in N \backslash\{0\} ; k=1, \ldots, K \\
& \quad r=1,2, \ldots . R \\
& U_{i k r}-U_{j k r}+\operatorname{Cap}_{k} X_{i j k r} \leq \operatorname{Cap} p_{k}-Q_{j} \forall i, j \in N \backslash\{0\} \\
& i \neq j, k=1, \ldots, K ; r=1, \ldots R \\
& \sum_{i=0}^{N} \sum_{j=0}^{N} \sum_{r=1}^{R} T_{i j} X_{i j k r} \leq \operatorname{Tmax} \quad \forall k=1, \ldots, K \\
& Y_{i k r} \in\{0,1\} \quad \forall i \in N ; k=1, \ldots, K, r=1, \ldots, R \\
& X_{i j k r} \in\{0,1\} \quad \forall i, j \in N ; k=1, \ldots, K, r=1, \ldots, R
\end{aligned}
$$

The objective function in (1) is to minimize the total cost of the trip, including vehicle fixed cost and vehicle variable cost. Constraints (2) ensure that every customer is visited only once. Constraints (3) show that the maximum number of routes and vehicles can be used. Constraints (4) and (5) show the same vehicle and route enters and leaves a given customer. Constraints (6) ensure that the demand of customer is the accumulation of volume unit of the product multiplied by order quantity of product. Constraints (7) make the sequence of the activated trip is from $R$ to $R+1$. Constraints (8) ensure that every trip does not exceed the vehicle capacity. Constraints (9) and (10) ensure there are no subtour. Constraint (11) is the restriction of a maximum working hour per day. Constraints (12) and (13) define the solution space of the decision variables.

\section{Model Testing}

To explain the model, we generated a small instance with 7 vertices and two types of vehicle. There are two vehicles type 1 (small capacity) and one vehicle type 2 (big capacity). Vertex 1 is denoted as a depot, and the others are denoted as a customer, and there are three types of vehicles. The maximum working hour per day is 420 minutes. This small instance is then tested using 2 kinds of scenarios.

The first scenario: The company own the vehicles (we consider the variable cost, only). The second scenario: The company own the vehicles, but the third party running the business. In this case, there will be a fixed-cost for renting the vehicles, which depend on the vehicles-type. Exact method branch and bound in solver LINGO 16.0 is used to solve this small instance. The customer demand data, travel time data from customer $i$ to $j$ and vehicle specification data for small instances testing are shown in Table 1, Table 2 and Table 3.
Table 1. Customer demand for small instance

\begin{tabular}{ccccccccc}
\hline \multirow{2}{*}{ Product Type } & \multirow{2}{*}{ Volume unit } & \multicolumn{1}{c}{ Customer demand } \\
\cline { 3 - 9 } & & 1 & 2 & 3 & 4 & 5 & 6 & 7 \\
\hline $\mathrm{A}$ & 2 & 0 & 0 & 5 & 0 & 5 & 0 & 10 \\
$\mathrm{~B}$ & 5 & 0 & 1 & 2 & 3 & 4 & 7 & 5 \\
$\mathrm{C}$ & 10 & 0 & 2 & 1 & 3 & 2 & 0 & 0 \\
\hline
\end{tabular}

Table 2. Travel time from customer $\mathrm{i}$ to $\mathrm{j}$ for small instances (in minutes)

\begin{tabular}{cccccccc}
\hline & 1 & 2 & 3 & 4 & 5 & 6 & 7 \\
\hline 1 & 0 & 13 & 13 & 13 & 16 & 42 & 64 \\
2 & 13 & 0 & 39 & 14 & 14 & 12 & 30 \\
3 & 13 & 39 & 0 & 7 & 14 & 5 & 8 \\
4 & 13 & 14 & 7 & 0 & 9 & 5 & 5 \\
5 & 16 & 14 & 14 & 9 & 0 & 21 & 69 \\
6 & 42 & 12 & 5 & 5 & 21 & 0 & 23 \\
7 & 64 & 30 & 8 & 5 & 69 & 23 & 0 \\
\hline
\end{tabular}

Table 3. Vehicle Specification

\begin{tabular}{cccc}
\hline Vehicle & Capacity & $\begin{array}{c}\text { Fixed cost per } \\
\text { vehicle }\end{array}$ & $\begin{array}{c}\text { Variable cost per } \\
\text { minute per vehicle }\end{array}$ \\
\hline 1 & 80 & 1000 & 200 \\
2 & 80 & 1000 & 200 \\
3 & 250 & 6000 & 700 \\
\hline
\end{tabular}

Table 4. Output for the first scenario

\begin{tabular}{ccccc}
\hline Vehicle & Route & Load & $\begin{array}{c}\text { Vehicle } \\
\text { Capacity }\end{array}$ & $\begin{array}{c}\text { Travelled } \\
\text { time }\end{array}$ \\
\hline 1 & $1(1-3-7-1)$ & 75 & 80 & 60 \\
& $2(1-6-4-1)$ & 80 & 80 & 43 \\
2 & $1(1-2-5-1)$ & 75 & 80 & 85 \\
\hline
\end{tabular}

Table 5. Output for the second scenario

\begin{tabular}{ccccc}
\hline Vehicle & Route & Load & $\begin{array}{c}\text { Vehicle } \\
\text { Capacity }\end{array}$ & $\begin{array}{c}\text { Travelled } \\
\text { time }\end{array}$ \\
\hline 2 & $1(1-6-4-1)$ & 80 & 80 & 60 \\
& $2(1-2-5-1)$ & 75 & 80 & 43 \\
& $3(1-3-7-1)$ & 75 & 80 & 85 \\
\hline
\end{tabular}

The instance above is then being solved based on two scenarios above using branch and bound in LINGO 16.0. The output for the first scenario and the second scenario is shown in Table 4 and Table 5.

The total cost for the first scenario is 37.600 and using two vehicles (vehicle 1 and vehicle 2). Table 4 shows that the output of the LINGO 16.0 is verified because the output is not exceeding the constraints on the model, so the mathematical model is also verified. If every route does not exceed the vehicle capacity, then the vehicles can make multi-trip. In this case, those vehicles can visit all customers exactly once. Moreover, the total time of each vehicle will not exceed the maximum working hour per day, i.e. 420 minutes.

The total cost for the first scenario is 38.600 and using only one vehicle (vehicle 2). The vehicle used in 

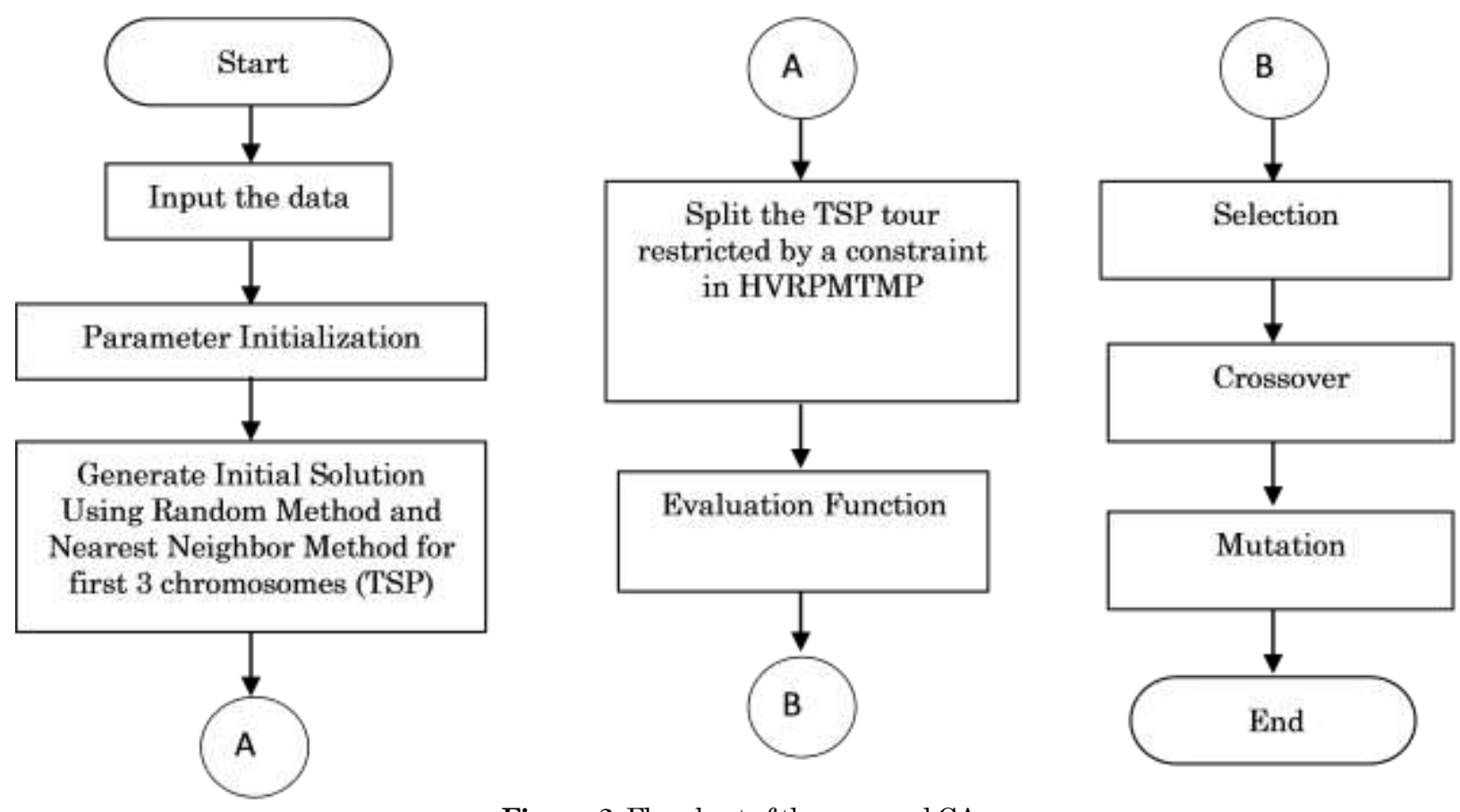

Figure 2. Flowchart of the proposed GA

scenario 2 is less than in scenario 1 because there is fixed cost in each vehicle so to minimize the total cost that includes fixed cost, the number of the vehicle used is also minimized. From Table 5 we know that the output of the mathematical model is verified because the output is not exceeding the constraints on the model, so the mathematical model is also verified. If every route does not exceed the vehicle capacity, then the vehicle can make a multi-trip. In this case, the vehicles can visit all customers exactly once. Moreover, the total time of each vehicle will not exceed the maximum working hour per day.

\section{Proposed Genetic Algorithm}

Because the nature of HVRPMT that is NP-hard problem, so in solving the large problem, using branch and bound is not efficient in term of computational time. We proposed a genetic algorithm (GA) for solving a large-real problem. We chose GA instead of the other metaheuristics since the GA performance is outstanding compared to others. Even if some other metaheuristics can find a better solution than GA, GA can generally find adequate solutions in a shorter time frame. Those are the main reasons why GA are still used in solving the routing, locating and other NP-hard problems. There have been many types of research done about solving VRP with GA where the researchers focused on the practical usage of the GA on VRP real-world problem (Karakatic and Podgorelec [20]). This section describes the details about the components of the proposed GA for this research. The flowchart of the proposed GA is shown in Figure 2.

\section{Solution Representation}

Encoding solution of the problem into a chromosome has a high impact on GA. Since the sequence of the customer must be different, we use a permutation encoding to make sure there is no same customer in every chromosome. The solution is represented by an array so that the values of the genes correspond to the nodes selected to form the collection of generalized routes while $\{1\}$ is a trip splitter on one vehicle and $\{0\}$ is a vehicle splitter. For example for 7

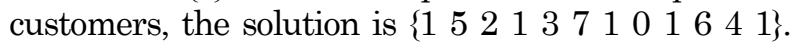
Route 1 is $1-5-2-1$ which is served by vehicle 1 on first route. Route 2 is 1-3-7-1 which is served by vehicle 1 on second route. Route 3 is 1-6-4-1 which is served by vehicle 2 on first route.

\section{Feasible Solution to Handling Constraints}

In this research, the only feasible solution is generated on every chromosome. This way to the only generated feasible solution is called preserving strategies (Talbi [21]). First, we use a sequence of a customer without trip splitter like in Traveling Salesman Problem (TSP) solution. This sequence is split by a constraint of vehicle capacity and working hour per day. We start from the first customer in the sequence and then move to the next customer while checking the vehicle capacity and working hour per day. If the load of the sequence exceeds the vehicle capacity but still not exceed working hour, then the same vehicle is used again until it exceeds a working hour. If the working hour is exceeded, then the next vehicle is used. This step is continued until all of the customers are served. The pseudocode to handling constraints is given in Figure 3 . 
1. Initialize index of the customer, index of the route, index of vehicle, a load of the route, the load of the vehicle, total load, duration of route, duration of the vehicle, total duration.

2. While index of vehicle $<=$ total number of vehicle

3. While index of route $<=$ total route

4. While index of customer $<=$ total number of customer and load of route $<=$ vehicle capacity and duration of route $<=$ working hour and index of vehicle $<=$ total number of vehicle load of route $=$ load of route + demand of customer $\mathrm{i}$

duration of route $=$ duration of route + time to visiting customer i from $\mathrm{i}-1$ based on the sequence of customer if load of route $>$ vehicle capacity

load of route $=$ load of route - demand of customer i

duration of route $=$ duration of route-time to visiting customer i- 1 to $\mathrm{i}$

end if vehicle than go back to depot

if duration of route $>$ working hour

load of route $=$ load of route - demand of customer $\mathrm{i}$

duration of route $=$ duration of route-time to visiting customer i- 1 to $\mathrm{i}$

end if

break while (3)

end while (3)

load of vehicle $=$ load of vehicle + demand of customer i

duration of vehicle $=$ duration of vehicle + duration of route-time to visiting customer i- 1 to $i$

if duration of vehicle $>$ working hour

load of vehicle $=$ load of vehicle - demand of customer i

break while (2)

end if

if index of cutomer $>$ number of customer

break while (2)

end if

end while (2)

cost $=$ fixed cost of vehicle + variable cost of vehicle*duration of vehicle

total load $=$ total load + load of vehicle

total duration $=$ total duration + duration of vehicle

if index of customer $>$ number of customer break while (1)

end if

Total cost $=$ Total cost + cost

end while (1)

Figure 3. Pseudocode to handling constraints

\section{Initial Population}

Initially, the population of travelling salesman problem (TSP) tour is constructed by following the Nearest Neighbor (NN) heuristic algorithm and random procedure. First of three chromosomes is constructed with the NN algorithm with a random initial customer. The rule of NN algorithm is always going to the next nearest unvisited customer until all customer is visited while the random procedure is constructed a random permutation of $n$ customer.

\section{Evaluation Function}

Every feasible chromosome is evaluated using its fitness value which measures its quality. The fitness value is given by the total cost of travelling all the vehicles, both fixed and variable vehicle cost. In a natural evolution, an individual with high fitness value will survive while an individual with low fitness value will die. GA is based on this survival-ofthe-fittest principle of nature, so it tries to maximize a function called the fitness function and naturally appropriate of solving maximization problem. For a maximization problem, the fitness function, $F(X)$, can be taken to be same as the objective function $f(X)$. For minimization problem, it can be transformed into a maximization problem first. The commonly used transformation to convert minimization problem to a fitness function is $F(X)=$ $1 / f(X)$ (Rao [22]). In this research, the objective function is to minimize the total cost than the fitness value of each chromosome is 1 / total cost.

\section{Selection}

In the GA, an appropriate method for choosing chromosomes for the crossover must be employed to give more chance to those chromosomes in a population that is the most fit. In this proposed GA, roulette wheel and linear rank selection method are used during the selection process. The roulette wheel is known as the best solution method (Gen and Chang [23]), and rank selection is commonly used in GA. 


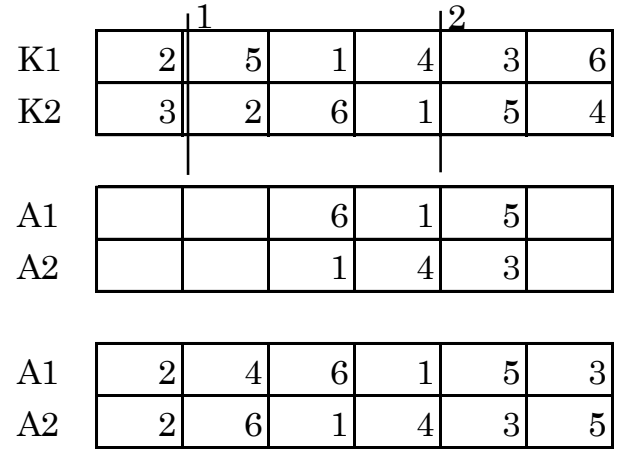

Figure 4. Scheme of order crossover

Table 6. Vehicle Specification

\begin{tabular}{cccc}
\hline Vehicle & $\begin{array}{c}\text { Capacity } \\
\left(\mathrm{cm}^{3}\right)\end{array}$ & $\begin{array}{c}\text { Fixed cost per } \\
\text { vehicle }(\mathrm{Rp})\end{array}$ & $\begin{array}{c}\text { Variable cost per } \\
\text { minute per vehicle } \\
(\mathrm{Rp})\end{array}$ \\
\hline 1 & 292384 & 70000 & 240.74 \\
2 & 292384 & 70000 & 240.74 \\
3 & 292384 & 70000 & 240.74 \\
4 & 5309436 & 200000 & 866.667 \\
\hline
\end{tabular}

\section{Crossover}

Through the selection method described above, two chromosomes from the current population are selected from the mating parents by means of the probability of crossover. If a randomly generated number between 0 and 1 is smaller than the probability of crossover, these two chromosomes reproduce to form new members to be included in the next generation. Otherwise, the crossover does not take place. In this proposed GA the order crossover is applied. In order crossover, one part of a chromosome is exchanged while maintaining a sequence of cities that are not part of the chromosome. The illustration of order crossover which applied in this research can be shown in Figure 4.

From Figure 5, initially, we generate two random cut points (1) and (2) to cut two parent chromosomes, K1 and K2. Then, two-child chromosomes, A1 and A2, get the genes from $\mathrm{K} 1$ and $\mathrm{K} 2$ chromosomes crosswise. A1 chromosome gets $\{6,1,5\}$ and A2 chromosome gets $\{1,4,3\}$. The empty genes position in A1 chromosome is filled by the K1 genes, sequentially from gen 1 to gen 6 , which is not yet in A1. The same fashion is done on chromosome A2.

\section{Mutation}

Mutation operator brings random changes into a single chromosome. If a randomly generated number between 0 and 1 is smaller than the probability of mutation, these chromosomes changes to form a new member in the next generation. Otherwise, the mutation does not take place. These random changes prevent premature local convergence. In this proposed GA, the mutation is done by choosing three different kinds of mutation procedures, according to Santosa and Ai [24]. The random number between 1 and 3 is generated to choose one of the mutation procedure. Suppose there is a route $4-6-\mid 2-3-5$ $-1 \mid-7$. Three different kinds of mutation procedures are as follows:

Flip: is done by flipping a segment of the route between two vertical lines to become $4-6$ - |1 - 5$3-2$ | -7 .

Swap: is done by swapping the customer at 3 and 6 to become $4-6-|1-3-5-2|-7$.

Slide: is done by sliding route between two vertical lines to become $4-6-|5-3-2-1|-7$.

\section{A Real Case Problem}

Real case in this research is a distribution problem in one of a pharmacy distribution company in Indonesia. The case is the company need to deliver its multi-products to its 55 customers using heterogeneous vehicles. It has two types of vehicles (see Table 6 for the vehicles specification). The variable cost includes fuel cost, which depended to vehicle type. Based on the data from the company, there are two types of vehicles (car and motorcycle) that is owned by that company to distribute its products. Vehicle 4 (car) can take a distance about 7,5 km per litre of fuel and vehicle 1, 2, 3 (motorcycle) can take a distance about $36 \mathrm{~km}$ per litre of fuel. The average velocity for vehicle 4 is $60 \mathrm{~km} /$ hour and vehicle $1,2,3$ are $80 \mathrm{~km} /$ hour. Fuel cost per litre is Rp 6,500, 00 . Fixed cost is obtained by the cost to rent a vehicle per day if the company does not own that vehicle. The calculation to get variable cost for every vehicle can be shown in Table 7.

In this real case, there are vehicles with small capacity that can perform several trips during its workday (vehicle 1,2 and 3). The vehicle variable cost includes the fuel cost per minute that is dependent on the vehicle type. Driver working hours per day are 7 hours (420 minutes).

This real case is then solved using two scenarios mention in the previous section.

Currently, the company grouped the customers based on their distance to the depot. If the distance from the depot is more than $20 \mathrm{~km}$ than this customers is served by vehicle 4 (car) and if the distance the depot is less than $20 \mathrm{~km}$ than those customers are served by vehicle 1,2 or 3 (motorcycle). The company allows the vehicle 1,2 and 3 (motorcycle) to travel more than one route to maximize its utilization. Then the route of each vehicle is determined 
Table 7. Calculation of fuel cost

\begin{tabular}{cccccc}
\hline Vehicle & $\begin{array}{c}\text { Fuel consumption } \\
(\mathrm{km} / \text { litre) }\end{array}$ & $\begin{array}{c}\text { Average velocity } \\
\text { (km/hour) }\end{array}$ & $\begin{array}{c}\text { Fuel consumption } \\
\text { conversion (litre/hour) }\end{array}$ & $\begin{array}{c}\text { Fuel consumption } \\
\text { conversion (litre/minute) }\end{array}$ & $\begin{array}{c}\text { Fuel cost } \\
\text { (Rp/minute) }\end{array}$ \\
\hline $1,2,3$ & 36 & 80 & 2.22 & 0.037 & 240.74 \\
4 & 7.5 & 60 & 8 & 0.133 & 866.667 \\
\hline
\end{tabular}

arbitrarily based on the driver experiences. The distribution route and the vehicle assigned to them are as follows

Vehicle 1 (motorcycle):

route $1: 1-11-31-14-45-48-1$

route $2: 1-54-53-44-55-52-1$

Vehicle 2 (motorcycle):

route 1: $1-49-47-38-29-32-35-51-1$

route $2: 1-56-4-3-36-43-46-1$

Vehicle 3 (motorcycle):

route $1: 1-50-42-41-39-21-40-1$

Vehicle 4 (car):

route $1: 1-2-33-34-37-30-9-10-5-6$

$-15-17-18-8-12-16-19-20-24-27-$ $25-23-26-22-28-7-13-1$

\section{Using an exact method to solve the real case} problem

In this section, the real case is solved using the exact method branch and bound in LINGO 16.0 solver. Because HVRPMTMP is NP-hard problem than we try with the small number of customer first to know how far branch and bound can handle the problem as the number of customers is getting bigger. If the exact method can not handle the real case in considerable computational time, then the genetic algorithm is used. Two scenarios like in small instance is also used in this real case problem. The result from LINGO 16.0 for the first scenario is shown in Table 8.

Table 8 shows that LINGO 16.0 can solve optimally within 10.800 seconds only for 8 customers. The computational time in LINGO 16.0 is increasing exponentially due to the number of customers are getting bigger. Figure 5 shows the computational time for LINGO 16.0.

The result from LINGO 16.0 for the second scenario is shown in Table 9.

From Table 9, we can know that LINGO 16.0 can solve optimally within 10.800 seconds only for 7 customers. The computational time in LINGO 16.0 is increasing exponentially due to the number of customers are getting bigger.

\section{Genetic Algorithm Verification}

Verification of the proposed GA is done by comparing the result of the proposed GA and result of the exact method by LINGO 16.0. Since LINGO 16.0 cannot solve the problem more than 9 customers after 10800 seconds than we use a GA for solving the big problem in more reasonable computational time. Comparison of the result between the proposed GA and LINGO 16.0 for two scenarios are shown in Table 10 and Table 11.

Table 8. LINGO 16.0 result for the first scenario in solving the real case problem

\begin{tabular}{cccc}
\hline $\begin{array}{c}\text { Number of } \\
\text { customers }\end{array}$ & Total cost & $\begin{array}{c}\text { Number of } \\
\text { vehicles used }\end{array}$ & $\begin{array}{c}\text { Computation } \\
\text { time (s) }\end{array}$ \\
\hline 5 & 19018.5 & 1 & 18 \\
6 & 24314.7 & 1 & 46 \\
7 & 40444.3 & 2 & 1265 \\
8 & 39722.1 & 2 & 3149 \\
9 & 54407.2 & 2 & $10800^{*}$ \\
\hline
\end{tabular}

*not solved optimally after $10800 \mathrm{~s}$

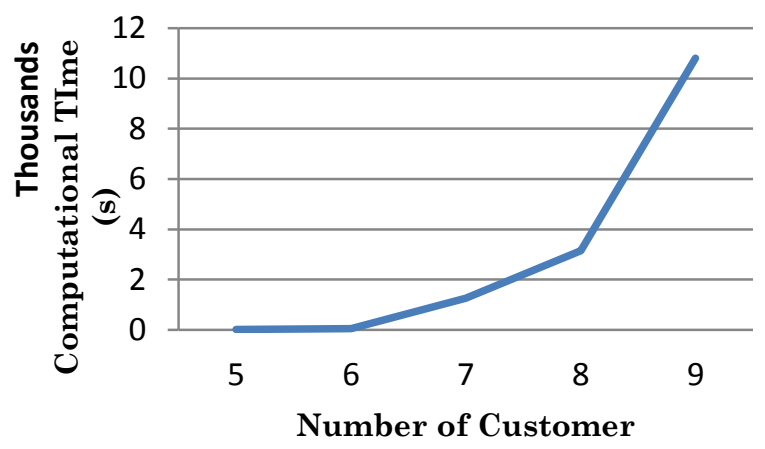

Figure 5. Computational time using LINGO 16.0 for the first scenario

Table 9. LINGO 16.0 result for the second scenario in solving the real case problem

\begin{tabular}{cccc}
\hline $\begin{array}{c}\text { Number of } \\
\text { customers }\end{array}$ & Total cost & $\begin{array}{c}\text { Number of } \\
\text { vehicles used }\end{array}$ & $\begin{array}{c}\text { Computation } \\
\text { time (s) }\end{array}$ \\
\hline 5 & 89018.5 & 1 & 32 \\
6 & 94314.7 & 1 & 166.7 \\
7 & 110444.3 & 1 & 3402.01 \\
8 & 109722.1 & 1 & $10800^{*}$ \\
9 & 124407.2 & 1 & $10800^{*}$ \\
\hline *not solved optimally after 10800 s &
\end{tabular}

Table 10. Comparison the result for the first scenario

\begin{tabular}{ccccc}
\hline \multirow{2}{*}{$\begin{array}{c}\text { Number of } \\
\text { customers }\end{array}$} & \multicolumn{2}{c}{ LINGO 16.0 } & \multicolumn{2}{c}{ Proposed GA } \\
\cline { 2 - 5 } & Total cost & $\begin{array}{c}\text { Computati } \\
\text { on time (s) }\end{array}$ & Total cost & $\begin{array}{c}\text { Computati } \\
\text { on time (s) }\end{array}$ \\
\hline 5 & 19018.5 & 18 & 19018.5 & 3.25 \\
6 & 24314.7 & 46 & 24314.7 & 4.39 \\
7 & 40444.3 & 1265 & 40444.3 & 4.4 \\
8 & 39722.1 & 3149 & 39722.1 & 4.4 \\
9 & 54407.2 & $10800^{*}$ & 54407.2 & 4.5 \\
\hline
\end{tabular}

*not solved optimally after $10800 \mathrm{~s}$ 
Table 11. Comparison of the result for the second scenario

\begin{tabular}{cccrc}
\hline $\begin{array}{c}\text { Number of } \\
\text { customers }\end{array}$ & \multicolumn{2}{c}{$\begin{array}{c}\text { LINGO 16.0 } \\
\text { Total cost } \begin{array}{c}\text { Computation } \\
\text { time (s) }\end{array}\end{array}$} & $\begin{array}{r}\text { Total cost } \\
\end{array}$ & \multicolumn{2}{c}{$\begin{array}{c}\text { Computati } \\
\text { on time (s) }\end{array}$} \\
\hline 5 & 89018.5 & 32 & 89018.5 & 3.44 \\
6 & 94314.7 & 166.7 & 94314.7 & 3.81 \\
7 & 110444.3 & 3402.01 & 110444.3 & 3.88 \\
8 & 109722.1 & $10800^{*}$ & 109722.1 & 4.07 \\
9 & 124407.2 & $10800^{*}$ & 124407.2 & 16 \\
\hline *not solved optimally after 10800 s
\end{tabular}

As shown in Table 10 and Table 11, we know that until 9 customers, GA can give the same solution as LINGO 16.0 solver but in a more reasonable time. By this result, the algorithm is verified and can be used to solve the real case with 55 customers.

\section{Parameter Setting}

The parameters of GA are population size, probability of crossover, probability of mutation and a maximum number of generation. The values of those parameters need to be selected carefully. A good parameter setting is essential for the metaheuristics algorithm like GA to obtain a good solution. In order to obtain proper parameters for GA, design experiments can be implemented to study the effects of the input factors and their interactions on the system performances and delineate which factor has the most effects to the responses (Shahsavar et al. [25]). Furthermore, when the study involves two or more factors, the factorial design is generally the most efficient way of delineating significant factors (Montgomery [26]). The effects of different patterns and parameters along with their interactions can be analyzed using a factorial design such as a full factorial design ( $2^{\mathrm{k}}$ factorial) in which $k$ is the number of factors (Shahsavar et al. [25]). To get a high level and low level to be used in this method, a pilot experiment is performed by determining the initial values for each parameter to be tested (Lathifah [27]). The pilot experiment started by

Table 12. One at A Time (OAT) method for parameter setting

\begin{tabular}{cccccc}
\hline No & Parameter & Population size & Maximum generation & Crossover probability & Mutation probability \\
\hline 1 & & 50 & 2000 & 0.8 & 0.001 \\
2 & \multirow{2}{*}{ Population size } & 100 & 2000 & 0.8 & 0.001 \\
3 & 150 & 2000 & 0.8 & 0.001 \\
4 & & 100 & 2000 & 0.8 & 0.001 \\
\hline 5 & & 100 & 1000 & 0.8 & 0.001 \\
6 & \multirow{2}{*}{ Maximum generation } & 100 & 1500 & 0.8 & 0.001 \\
7 & & 100 & 2000 & 0.8 & 0.001 \\
8 & & 100 & 2500 & 0.8 & 0.001 \\
\hline 9 & & 100 & 2000 & 0.65 & 0.001 \\
10 & Crossover probability & 100 & 2000 & 0.7 & 0.001 \\
11 & & 100 & 2000 & 0.8 & 0.001 \\
12 & & 100 & 2000 & 0.9 & 0.001 \\
\hline 13 & & 2000 & 0.8 & 0.001 \\
14 & Mutation probability & 100 & 2000 & 0.8 & 0.005 \\
15 & & 100 & 2000 & 0.8 & 0.01 \\
16 & & 100 & & 0.8 & 0.05 \\
\hline
\end{tabular}

Table 13. The result of OAT method

\begin{tabular}{|c|c|c|c|c|c|c|c|c|}
\hline No & Replication 1 & Replication 2 & Replication 3 & Replication 4 & Replication 5 & Average & High level & Low level \\
\hline 1 & 215943.78 & 217147.48 & 220277.1 & 195721.62 & 297410.898 & 229300.18 & & \\
\hline 2 & 209433.8 & 231832.62 & 194999.4 & 210888.24 & 203666.04 & 210164.02 & V & \\
\hline 3 & 221962.28 & 227258.56 & 213777.12 & 218832.66 & 211610.46 & 218688.22 & & V \\
\hline 4 & 219554.88 & 225332.64 & 230388.18 & 225332.64 & 230388.18 & 226199.3 & & \\
\hline 5 & 228943.74 & 212332.68 & 230388.18 & 212091.94 & 226295.6 & 222010.43 & & \\
\hline 6 & 203906.78 & 218351.18 & 221962.28 & 220517.84 & 218591.92 & 216666 & & \\
\hline 7 & 209433.8 & 231832.62 & 194999.4 & 210888.24 & 203666.04 & 210164.02 & & V \\
\hline 8 & 220999.32 & 196684.58 & 216425.26 & 228462.26 & 184888.32 & 209491.95 & V & \\
\hline 9 & 213536.38 & 203425.3 & 196684.58 & 218832.66 & 219554.88 & 210406.76 & & \\
\hline 10 & 200054.94 & 214017.86 & 199573.46 & 205110.48 & 220036.36 & 207758.62 & V & \\
\hline 11 & 209433.8 & 231832.62 & 194999.4 & 210888.24 & 203666.04 & 210164.02 & & V \\
\hline 12 & 215221.56 & 216184.52 & 208962.32 & 230388.18 & 227258.56 & 219603.3 & & \\
\hline 13 & 209433.8 & 231832.62 & 194999.4 & 210888.24 & 203666.04 & 210164.02 & & \\
\hline 14 & 206554.92 & 212091.94 & 222925.24 & 182480.92 & 222443.76 & 209299.36 & V & \\
\hline 15 & 227980.78 & 215943.78 & 206554.92 & 204147.52 & 204869.74 & 211899.35 & & \\
\hline 16 & 208240.1 & 212814.16 & 207517.88 & 213777.12 & 204629 & 209395.65 & & V \\
\hline
\end{tabular}


determining the initial values for each parameter to be tested. The initial values are based on the recommended values in the literature. We use the initial value suggested by De Jong [28]. The initial value of population size is 100 , the probability of crossover is 0.8 , the probability of mutation is 0.001 , and the maximum of generation is 2000 . Then we were using a One at A Time (OAT) method to get the low and high level for $2^{\mathrm{k}}$ factorial design in Table 12 and 13. For every parameter, we try some values that are set arbitrary based on the initial value. In every OAT experiment, we do replication for 5 times.

Based on the result in Table 12, we have 16 combinations in the parameter setting to get the high and low value for $2^{\mathrm{k}}$ factorial design. We do 5 times replication for each combination. The result of OAT can be shown in Table 13, and the result of high level and low level for $2^{\mathrm{k}}$ factorial design can be shown in Table 14.

As shown in Table 13, for each parameter, we set the best result as high level and the second best as low level. The total combination is 16 ( 2 total number of parameters $=2^{4}$ ). In $2^{\mathrm{k}}$ factorial design, each parameter combination is evaluated by doing 5 times replication in order to get the best combination of them. The combinations are shown in Table 15.

Table 14. The high level and low level of the proposed GA parameter

\begin{tabular}{ccc}
\hline Parameter & High level & Low level \\
\hline Population size & 100 & 150 \\
Maximum Generation & 2500 & 2000 \\
Crossover probability & 0.7 & 0.8 \\
Mutation probability & 0.005 & 0.05 \\
\hline
\end{tabular}

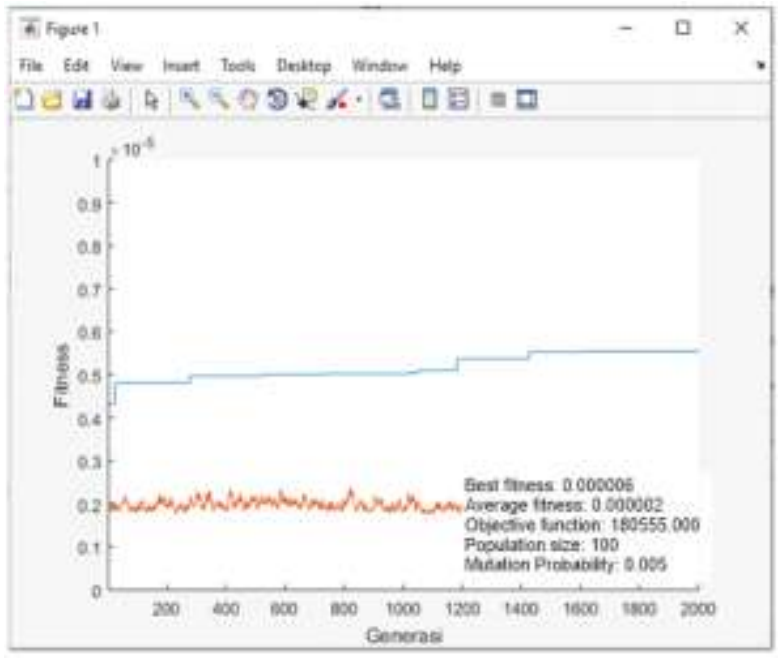

Figure 6. Graph of the result from MATLAB

The lowest average for all combinations is chosen as the best parameter combination and then is applied to proposed GA. The best parameter combination is population size $=100$, probability of crossover $=0.8$, probability of mutation $=0.005$ and the maximum of generation $=2000$.

\section{Using the Proposed Genetic Algorithm to Solve the Real Case Problem}

After parameter setting for the proposed genetic algorithm is done and because the exact method can not solve the real case optimally in reasonable computational time, then we use the proposed genetic algorithm to solve it. The proposed GA is run in MATLAB R2015a. There are also two scenarios, like in small instances. Figure 6 shows the graph of the result from MATLAB for the first scenario.

Table 15. $2^{\mathrm{k}}$ factorial design combination for proposed GA

\begin{tabular}{cccccc}
\hline No & Population size & Maximum generation & Crossover probability & Mutation probability & Average Result \\
\hline 1 & 100 & 2000 & 0.7 & 0.005 & 212573.4 \\
2 & 100 & 2000 & 0.7 & 0.05 & 213215.4 \\
3 & 100 & 2000 & 0.8 & 0.005 & $189622.9^{*}$ \\
4 & 100 & 2000 & 0.8 & 0.05 & 213696.9 \\
5 & 100 & 2500 & 0.7 & 0.005 & 202061.1 \\
6 & 100 & 2500 & 0.7 & 0.05 & 209524 \\
7 & 100 & 2500 & 0.8 & 0.005 & 198931.5 \\
8 & 100 & 2000 & 0.8 & 0.05 & 208641.3 \\
9 & 150 & 2000 & 0.7 & 0.005 & 222764.7 \\
10 & 150 & 2000 & 0.7 & 0.05 & 216104.3 \\
11 & 150 & 2000 & 0.8 & 0.005 & 201098.1 \\
12 & 150 & 2500 & 0.8 & 0.05 & 215462.3 \\
13 & 150 & 2500 & 0.7 & 0.005 & 215943.8 \\
14 & 150 & 2500 & 0.7 & 0.05 & 218190.7 \\
15 & 150 & 2500 & 0.8 & 0.005 & 206956.2 \\
16 & 150 & & 0.8 & 0.05 & 209684.5 \\
\hline
\end{tabular}

*best combination of proposed GA parameter 
From Figure 6 we know that the minimum cost obtained is 180.555 (Objective function). The result of the first scenario obtained can be seen as follows Vehicle 1 (motorcycle):

route 1 : $1-13-8-12-16-1$

route 2 : $1-4-37-5-30-1$

route $3: 1-48-45-31-46-43-36-9-15-1$

route $4: 1-38-6-3-1$

route $5: 1-34-10-22-11-1$

Vehicle 2 (motorcycle):

route $1: 1-52-28-19-25-24-23-26-40-$ $21-1$

route $2: 1-49-2-47-29-51-1$

route 3 : $1-54-27-44-55-53-56-1$

route $4: 1-14-17-18-20-7-35-1$

Vehicle 3 (motorcycle):

route $1: 1-50-39-41-42-32-33-1$

The result obtained is only used vehicle 1, 2 and 3 (motorcycle) because the variable cost of the motorcycle is smaller than the variable cost of a car (vehicle 4). Since vehicles 1,2 , and 3 can still distribute the products to customers without violating capacity and working hour restrictions, then the proposed GA is not including the vehicle 4 in order to minimize total cost. Then, we check this result whether violating capacity and working hour constraint that is shown in Table 16 below. It shows that every vehicle on every route does not violate capacity constraint, and every vehicle does not violate working hour per day that is 420 minutes.

While the result for the second scenario can be seen in Table 17 and as follows

Vehicle 1 (motorcycle):

route $1: 1-13-8-12-16-1$

route 2 : $1-4-37-5-30-1$

route $3: 1-48-45-31-46-43-36-9-15-1$

route $4: 1-38-6-3-1$

route 5 : $1-34-10-22-11-1$

route $6: 1-50-39-41-42-32-33-1$

Vehicle 2 (motorcycle):

route $1: 1-52-28-19-25-24-23-26-40-$ $21-1$

route $2: 1-49-2-47-29-51-1$

route $3: 1-54-27-44-55-53-56-1$

route 4 : $1-14-17-18-20-7-35-1$

In the second scenario we assume that the company does not have any vehicles so it must rent the vehicle. The cost of rent is expressed by fixed cost. The vehicle used in the second scenario is fewer than the vehicle used in the first scenario because the objective is to minimize total fixed and variable cost so by using the fewer vehicle, the lower the fixed cost. The vehicle can be assigned more tour as long as the total delivery time does not violate the working hour restriction.

\section{Comparing the Result of the Proposed GA with the Company Decision}

Since the company in real case problem own their vehicle to distribute their products than only the result from the first scenario (only considering the variable cost of the vehicle) is compared to the distributional decision that is made by the company. The result from the proposed GA can reduce the

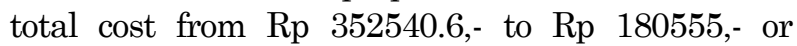
$48.78 \%$ from the company decision. This happens because, in the company policy, customers are clustered first based on the vehicle type, so the vehicle type with the high variable cost (vehicle $4 /$ car) is also used. In this research, vehicle 4 (car) is not used because the vehicles with low variable cost (motorcycle) are used maximally in their working day, and they can cover all the customer demand.

\section{Conclusion}

In this paper, a mathematical model for heterogeneous vehicle routing problem with multi-trips and multi-products (HVRPMTMP) with fixed and variable vehicle cost has been developed using developed four-index vehicle flow formulation. This model can be used generally in the same context of the distribution problem. Branch and bound is also used to solve a real case with 55 customers using LINGO 16.0. The real case can be solved optimally within 10,800 seconds with LINGO 16.0 until 8 customers.

The result from the proposed GA is then compared to the result from LINGO 16.0 until 9 customers, and both the result is same so GA is verified and can be used to solve the real case with 55 customers. The result of the proposed GA can reduce the total cost from $\operatorname{Rp} 352540.6$ to $\mathrm{Rp} 180555$ or $48.78 \%$ from the current company decision. In term of fixed cost depending on the ownership status of the vehicle, if the company owns the vehicles, then there is no difference whether using fewer vehicles or not as long as the vehicle types are same (same variable cost). If the company does not own the vehicles, then there is a difference using fewer vehicles because there is an effort required to provide a vehicle (the cost to rent vehicles that are associated as fixed cost).

The company can apply our suggested route and vehicle used based on the result of our proposed GA to minimize total distributional cost. It means that the car is not used as long as the number of customers does not change. There are advantages and disadvantage that the company does not use the car. The advantages are the company does not need the driver for the car, and since the car is not used, the company does not need to spend a lot of money 
Table 16. The result of the proposed genetic algorithm for the first scenario

\begin{tabular}{cccccc}
\hline Vehicle & Route & $\begin{array}{c}\text { Total capacity used per } \\
\text { route }\left(\mathrm{cm}^{3}\right)\end{array}$ & $\begin{array}{c}\text { Total capacity per } \\
\text { vehicle }\left(\mathrm{cm}^{3}\right)\end{array}$ & $\begin{array}{c}\text { Total time per route } \\
(\text { minutes })\end{array}$ & $\begin{array}{c}\text { Total time per vehicle } \\
(\text { minutes })\end{array}$ \\
\hline 1 & 1 & 282594.77 & 292384 & 60 & 310 \\
& 2 & 268423.41 & 292384 & 37 & \\
& 3 & 290517.3 & 292384 & 57 & 394 \\
& 4 & 253998.3 & 292384 & 34 & \\
\hline 2 & 5 & 276932.1 & 292384 & 212 & 46 \\
\hline
\end{tabular}

Table 17. The result of the proposed genetic algorithm for the second scenario

\begin{tabular}{ccccc}
\hline Vehicle & Route & Variable cost (Rp) & Fixed cost (Rp) & Total cost (Rp) \\
\hline 1 & 1 & 85703.44 & 70000 & \\
& 2 & & & \\
& 3 & & & \\
4 & 555703.4 \\
& 6 & & 70000 & 164851.6 \\
& 1 & 94851.56 & & \\
\hline 2 & 2 & & & 320555 \\
\hline
\end{tabular}

on maintenance. The disadvantage is if the company already have a driver for the car, then the company must consider the other job/task for the driver. Our approach is effective if the company does not have any vehicle for distributing the product since our approach can minimize the number of vehicles that it can minimize the fixed cost.

For further research, to enrich the type of the problem, it can be relevant by adding a constraint that small vehicle is used access the customer in the restricted area or to reduce the congestion in city logistics context that only use a small vehicle in the city center.

\section{References}

1. Braekers, K., Ramaekers, K., Nieuwenhuyse, I.V., The Vehicle Routing Problem: State of the Art Classification and Review. Computers \& Industrial Engineering, 99, 2015, pp. 300-313.

2. Toth, P., and Vigo D., Vehicle Routing: Problems, Methods, and Applications, 2014, Mathematical Optimization Society and Society for Industrial and Applied Mathematics (MOS-SIAM) Series in Optimization, Philadelphia.

3. Dantzig, G.B, and Ramser, J.H., The Truck Dispatching Problem. Management Science, 6, 1959, pp. 80-91.
4. Baldacci, R., Battara, M. and Vigo, D., Routing a Heterogeneous Fleet of Vehicles. The Vehicle Routing Problem: Latest Advances and New Challenges, 2008, pp. 1-25.

5. Cordeau, J-F., Laporte, G., Savelsbergh, M.W.P., Vigo, D., Barnhart, C. and Laporte, G. Vehicle Routing. Handbook in Operational Research and Management Science, 14, 2007, pp. 367-428.

6. Laporte, G., Fifty Years of Vehicle Routing, Transportation Science, 43(4), 2009, pp. 408-416.

7. Golden, B., Raghavan, S., and Wasil, E., The Vehicle Routing Problem: Latest Advances and New Challenges, Operations Research/Computer Science Interfaces Series, 43, Springer, 2008.

8. Koç, C., Bektas, T., Jabali, O., and Laporte, G., Thirty Years of Heterogeneous Vehicle Routing. European Journal of Operational Research, 2015, pp. 1-21.

9. Gendreau, M., Potvin, J.Y., Braysy, O., Hasle, G. and Lokketangen, A., Metaheuristic for the Vehicle Routing Problem and its Extension: A Categorized Bibliography. CIRRELT, 2007.

10. Suprayogi, and Priyandari, Y., Tabu Search for the Vehicle Routing Problem with Multiple Trips, Time Windows and Simultaneous Delivery-Pickup. Jurnal Teknik Industri, 19(2), 2017, pp 75-82.

11. Taillard, E.D., Laporte, G., and Gendreau, M., Vehicle Routing with Multiple Use of Vehicles. Journal of the Operational Research Society, 47 (8), 1996, pp. 1065-1070. 
12. Huang, C, and Lee, C.M., A Study of Multi-Trip Vehicle Routing Problem and Distribution Centre Location Problem. POMS 22 ${ }^{\text {nd }}$ Annual Conference, 2011, Nevada, USA.

13. Cattaruza, D., Absi, N., Feillet, D., and Vidal, T., A Memetic Algorithm for the Multi Trip Vehicle Routing Problem, European Journal of Operational Research, 236, 2014, pp. 833-848.

14. Prins, C., Efficient Heuristics for the Heterogeneous Fleet Multitrip VRP with Application to a Large-Scale Real Case. Journal of Mathematical Modelling and Algorithms, 1, 2002, pp. 135150.

15. Sexias, M.P. and Mendes, A.B., Column Generation for a Multitrip Vehicle Routing Problem with Time Windows, Drivers Work Hours and Heterogenous Fleet. Mathematical Problems in Engineering, 2013, pp. 1-13.

16. Cruz, J.C., Grasas, A., Ramalhinho, H., and Juan, A.A., A Saving-Based Randomized Heuristic for the Heterogeneous Fixed Fleet Vehicle Routing Problem with Multi-Trips. Journal of Applied Operational Research, 2014, pp. 69-81.

17. Coelho, V.N., Grasas, A., Ramalhinho, H., Coelho, I.M., Souza, M.J.F., Cruz, R.C., An ILSbased Algorithm to Solve a Large-Scale Real Heterogeneous Fleet VRP with Multi-Trips and Docking Constraints, European Journal of Operational Research, 250, 2016 pp. 367-376.

18. Prive, J., Renaud, J., Boctor, F. and Laporte, G., Solving a Vehicle Routing Problem Arising in Soft Drink Distribution. Journal of Operational Research Society, 2005.

19. De la Cruz, J.J., Paternina-Arboleda, C.D., Cantillo, V., and Montoya-Torres, J.R., A TwoPheromone Trail Ant Colony System-Tabu
Search Approach for the Heterogeneous Vehicle Routing Problem with Time Windows and Multiple Products. Journal of Heuristics, 19, 2013, pp. 233-252.

20. Karakatic S., and Podgorelec, V., A Survey of Genetic Algorithm for Solving Multi Depot Vehicle Routing Problem. Applied Soft Computing, 27, 2015, pp 519-532.

21. Talbi, E-G., Metaheuristic: From Design to Implementation, 2009, John Wiley \& Sons, New Jersey.

22. Rao, S.S., Engineering Optimization: Theory and Practice, $4^{\text {th }}$ edition. John Wiley \& Sons, Inc, 2009.

23. Gen, M, and Cheng, R., Genetic Algorithm and Engineering Optimization, 2007, John Wiley \& Sons, Inc.

24. Santosa, B and Ai, T.J., Pengantar Metaheuristik: Implementasi dengan Matlab, ITS Tekno Sains, 2018, Surabaya.

25. Shahsavar, M., Najafi, A.A, and Niaki, S.T.A., Statistical Design of Genetic Algorithm for Combinatorial Optimization Problems, Mathematical Problems in Engineering, 2011.

26. Montgomery, D.C., Design and Analysis of Experiments, 8th $^{\text {th }}$ edition, 2013, John Wiley \& Sons, New York.

27. Lathifah, A., Aplikasi Simulated Annealing Heuristik pada Permasalahan Heterogeneous Fleet Polution Routing Problem, Thesis in Master Program of Industrial Engineering Universitas Gadjah Mada, 2015, Yogyakarta.

28. De Jong, K.A., An Analysis of the Behavior of a Class of Genetic Adaptive Systems. Doctoral dissertation, University of Michigan, 1975. 